\title{
La selva e il colle. La ermeneutica dei generi nel primo canto dell'Inferno
}

Raffaele Pinto

Universitat de Barcelona

\begin{abstract}
L'articolo legge il primo canto dell' Inferno secondo la prospettiva della ermeneutica dei generi sessuali. La iniziale contrapposizione, che criminalizza il femminile come genere basso e negativo nella linea della misoginia antica e medievale, si trasforma, nella seconda parte del canto, in una visione modernamente complementare dei generi, nella quale il femminile viene anzi ridefinito come categoria culturalmente alta e positiva.
\end{abstract}

Parole chiave: Dante, Inferno I, studi di genere, maschile e femminile.

\section{Abstract}

This article reads the first canto of Inferno from the perspective of gender hermenutics. The initial opposition, which blames the female as the inferior and negative gender, in the line of ancient and medieval misogyny, transforms itself, in the second part of the canto, into a modern and complementary vision of genders, in that the female is indeed redefined as a high and positive cultural category.

Key words: Dante, Inferno I, gender studies, male and female.

Nella critica femminista la nozione di genere sessuale designa normalmente procedimenti espressivi e forme ideologiche legati alla rappresentazione della identità. ${ }^{1}$ Femminile e maschile, però, sono anche, nelle civiltà antiche, i poli ideali di un sistema di produzione simbolica su cui gravitano da una parte il pensiero mitico-religioso, con i suoi binarismi, ${ }^{2}$ dall'altra la struttura economico-sociale, con le sue gerarchie. Il simbolismo dei generi, nella storia delle mentalità,

1. Intendo «identità» come segno della differenza sessuale (sono però cosciente del fatto che il dibattito interno al femminismo su questo concetto è molto aspro -cfr., per un riepilogo, Rosi BraidotTI, Soggetto nomade. Femminismo e crisi della modernità, Roma: Donzelli Editore, 1995 [1994], p. 65-79).

2. Sul tema nella antichità giudeo-cristiana, cfr. Gian Luigi PRATO (a cura di), Ricerche storico bibliche, 1994, 1-2, Miti di origine, miti di caduta e presenza del femminino nella loro evoluzione interpretativa, XXXII settimana biblica nazionale. 
mostra in effetti uno slittamento dal significato politico dominante nelle società antiche a quello personale prevalente nelle società moderne. Indipendentemente dalle modalità e le fasi di tale slittamento, la cui ricostruzione storiografica è forse compito della nuova critica letteraria, si può verosimilmente affermare che esso è parallelo all'affioramento, nei testi, di forme autografiche di scrittura: il «maschile» rilevabile nell'epica dell'Iliade o della Chançon de Roland afferisce ad un simbolismo politico-religioso i cui valori (la giustizia, la forza, il coraggio etc.) configurano l'identità di tutta una classe sociale, l'ordine dei nobili o dei guerrieri; mentre il «maschile» rilevabile nella lirica di Petrarca o di Baudelaire rientra in un simbolismo morale e soggettivo, il cui valore egemonico è il desiderio. A prescindere dalle eccezioni che attenuerebbero tale divaricazione (scritture dell'io nell'antichità, ideologie sessuali collettive nella modernità; stratificazioni sincroniche dei due simbolismi, attivi su livelli differenti dello stesso testo), è chiaro che la distinzione «antico-moderno» è non solo pertinente alla opposizione «femminile-maschile», ma anche logicamente prioritaria, poiché è all'interno di tali modelli globali di civiltà (antichità — modernità) che gli astratti concetti generici si incarnano in simboli letterariamente produttivi. ${ }^{3}$

Da uno scrittore di frontiera fra antico e moderno come è Dante, e in mancanza di anteriori sondaggi sistematici sul simbolismo sessuale presente nella sua opera, è prudente attendersi indizi di genere afferenti ai due sistemi: valori archetipici «antichi» da una parte, contenuti personali «moderni» dall'altra. L'esperimento che qui si propone, cioè la lettura della Commedia (metonimicamente rappresentata dal canto proemiale $)^{4}$ secondo i concetti di genere, parte quindi senzaltro dal preliminare e metodologico ancoraggio alle categorie che strutturano i campi concettuali del femminile e del maschile sul piano antropologico generale; e l'analisi consisterà nel reperimento, ove possibile, di tali categorie nella lettera del testo, o, più esattamente, nelle sue figure. Ma si seguiranno anche percorsi di lettura autografici, alla ricerca della singolarità dantesca dell'idea di genere, ovviamente connessa alla fattuale circostanza che l'io che scrive si enuncia come un io maschile. Ė inevitabile che un tale approccio trascuri, in qualche misura, il piano del figurato, il vero che il velo delle figure occulta, così familiare ai lettori della Commedia, soprattutto in questo canto.

3. L'idea di "patriarcato» come categoria storica trasversale che neutralizza la distinzione antico-moderno, efficace forse sul piano politico-rivendicativo, è inutilizzabile sul piano teorico, perché impedisce di cogliere l'essenziale: l'affiorare, storicamente, di un soggetto genericamente (auto)determinato secondo la differenza sessuale (che è ovviamente incompatibile con una mascolinità imposta come universalistica legge del padre). L'errore di prospettiva dipende dal fatto che gli studi relativi al genere sessuale (femministi, soprattutto) hanno privilegiato, come universo concettuale di riferimento, la tradizione del pensiero filosofico, che conserva le categorie ideali antiche molto più di quanto non conservi le categorie espressive antiche la letteratura di tipo poetico: mentre il soggetto che conosce, in Cartesio e Platone, è identico, il soggetto che si esprime in Shakespeare è indeducibile da quello che si esprime in Sofocle.

4. Sulla funzione proemiale del primo canto, cfr. Antonino PAgLiaro, Ulisse. Ricerche semantiche sulla Divina Commedia, Messina-Firenze: D’Anna, 1967, p. 2-3. 
Ma il radicale mutamento di prospettiva critica dovrebbe compensare il sacrificio con un incremento di informazione forse non trascurabile.

Predicati del femminile sono, sul piano simbolico, la luna, il basso, la sinistra, la notte, il buio, lo scuro, il pesante, il rovescio, il freddo, l'umido, l'inferiore; predicati del maschile sono invece il sole, l'alto, la destra, il giorno, la luce, il chiaro, il leggero, il diritto, il caldo, il secco, il superiore. ${ }^{5}$

Le figure iniziali, «selva oscura» / "diritta via», oppongono subito i generi con i due aggettivi: oscura $=$ femminile $/$ diritta $=$ maschile. ${ }^{6}$ Un simbolismo più allusivo traspare dai significanti cammin, via, per l'idea maschile di movimento ordinato in uno spazio aperto, e selva, per l'idea femminile di reclusione (o movimento disordinato) in uno spazio chiuso. Ovvî infine, cioè allineati con il simbolismo convenzionale, sono i valori negativo del femminile e positivo del maschile: ${ }^{7}$
Nel mezzo del cammin di nostra vita
mi ritrovai per una selva oscura,
ché la diritta via era smarrita. ${ }^{8}$

5. Cfr. Françoise HérITIERE, Maschile/femminile, in Enciclopedia, Torino: Einaudi, vol. 8, p. $797-$ 812 (cfr. in particolare le p. 802-803). La studiosa rileva la presenza di queste dicotomie anche nel pensiero greco: qui «le categorie principali sono quelle del caldo e del freddo, del secco e dell'umido, che sono direttamente associate alla mascolinità (il caldo e il secco) e alla femminilità (il freddo e l'umido)... Nell'ordine del corpo, il caldo e l'umido sono dalla parte della vita, della gioia, del benessere..., il secco e il freddo sono dalla parte della morte... Il maschio è caldo e secco, associato al fuoco e al valore positivo, la femmina è fredda, umida, associata all'acqua e al valore negativo» (l'autrice ritorna più diffusamente sul tema in Maschile e femminile. Il pensiero della differenza, Roma-Bari: Laterza, 1997 [1996]).

6. Sulla selva, cfr. Eugenio RaGNI, v. selva, in Enciclopedia Dantesca, Roma: 1970-1978, vol. V, p. 137-142. Raccolgo qui due indicazioni: la lettura di Benvenuto da Imola, che interpreta la selva come allusione a "aliis mulieribus» (in riferimento a Purg., XXX, 126: «questi si tolse a me e diessi altrui»); e il significato di materia corporea e caos che il termine silva ha nella cosmologia neoplatonica (la hyle greca). All'interno di tale tradizione, indipendentemente dalla conoscenza che poteva averne Dante, vale la pena di ricordare che nella cosmogonia manichea la hyle, principio del male associato alla concupiscentia (epitymia, o edoné), veniva rappresentata come una divinità arimanica femminile: $A z$ (cfr. Antonio PANAINO, Figure femminili divine e demoniache nell'Iran antico, in «Ricerche storico bibliche...», p. 64-65).

7. Esiste, naturalmente, anche una femminilità positiva, nella cultura antica, vincolata all'idea della maternità, la cui simbologia è ben presente nel pensiero mitico-religioso- cfr., al riguardo, Erich Neumann, La Grande Madre. Fenomenologia delle configurazioni femminili dell'inconscio, Roma, 1981 [1956]. Una eco di tale complesso mitico, e delle relative mediazioni letterarie, è stata osservata nel personaggio dantesco di Matelda -cfr. Rosetta Migliorini Fissi, Da Matelda a Beatrice (cenni sull'archetipo del femminile), in Maria PICCHIO Simonelli (a cura di), Beatrice nell'opera di Dante e nella memoria europea, 12901990, «Atti del Convegno Internazionale. 10-14 dicembre 1990», Firenze-Napoli: Istituto Universitario Orientale, 1994, p. 183-206.

8. L'età di 35 anni come la metà di una vita umana che si sviluppi in modo naturale viene discussa in Convivio, IV, xxiii, 9: se si compara l'esistenza con un arco, «lo punto sommo... io credo che ne li perfettamente naturati sia nel trentacinquesimo anno". Tale "punto sommo» rappresenta la migliore combinazione delle quattro qualità umorali (caldo, freddo, 
Bisogna tener conto del fatto che il simbolismo femminile della selva e dello smarrimento era perfettamente codificato nella tradizione prossima a Dante, che ne trovava esempi nel Tesoretto di Brunetto Latini (il narratore, intristito dal pensiero delle guerre di fazione, a Firenze, si smarrisce in una «selva diversa» nella quale trova una donna che gli si rivela essere la Natura) e nel Detto del Gatto Lupesco (il narratore racconta di smarrire il cammino, «d' un amor ... pensando", e di ritrovarsi in un "diserto aspro e duro» in cui gli appaiono un gran numero di animali). I due testi hanno poi in comune un elemento che ci fa capire meglio la disposizione morale che Dante descrive con l'immagine iniziale del Poema. In entrambi il protagonista-narratore cammina «a capo chino»: «e io, in tal corrotto / pensando a capo chino, / perdei il gran cammino, / e tenni la traversa / d'una selva diversa» (Tesoretto, 186-190); «così m’andava l'altra dia / per un cammino trastullando / e d'un amor gia pensando / e andava a capo chino" (Detto, 4-7). Nel sonetto Cavalcando l'altrier per un cammino, commentato nel capitolo IX della Vita Nuova, Dante utilizza la stessa immagine per descrivere l'atteggiamento del personaggio di Amore (alter ego del poeta), che gli comunica la fine della sua relazione con la prima donna-schermo:
Cavalcando l'altr'ier per un cammino, pensoso de l'andar che mi sgradia, trovai Amore in mezzo de la via in abito leggier di peregrino.
Ne la sembianza mi parea meschino, come avesse perduto segnoria;
e sospirando pensoso venia, per non veder la gente a capo chino...

In tutti e tre i testi causa dello smarrimento è una sospensione del rapporto con il reale prodotta da una passione dell'anima che attrae violentemente a sé l'attenzione della mente. L'io viene pervertito (cioè sviato) dall'oggetto interno che assorbe i suoi pensieri. Tale oggetto è simbolicamente (oltre che lette-

secco, umido), giacché «la Gioventute», seconda delle quattro età dell'uomo (fra i 25 e i 45 anni), «'sappropria al caldo e al secco [cioè al maschile]» (ibid., 13)-sulle fonti medico-filosofiche di Dante e sulla discussione relativa, Cfr. C. Vasoli (a cura di), Convivio, Ricciardi, Milano-Napoli, 1988, p. 798 e sgg. Si consideri poi che nel capitolo seguente (XXIV, 12), a proposito della necessità che hanno gli adolescenti della guida dei loro maggiori, l'autore paragona la vita ad una città ignota: «sì come quello che mai non fosse stato in una cittade, non saprebbe tenere le vie, senza insegnamento di colui che l'hae usata; così l'adolescente, che entra ne la selva erronea di questa vita, non saprebbe tenere lo buono camino, se da li suoi maggiori non li fosse mostrato». Qui il simbolo della selva è già inserito in una trama concettuale che collega la vita alle vie e al cammino da una parte, e all'insegnamento di guide dall'altra: «li soi maggiori». Le risonanze scritturali (Isaia, 38, 10: «in dimidio dierum meorum vadam ad portas Inferi») della prima terzina della Commedia si sovrappongono ad un nucleo concettuale già acquisito, simbolico da una parte e scientifico dall'altra; più in generale, il profetismo della Commedia è molto più un registro di stile, accanto a tutti gli altri, che l'a priori dell'espressione poetica. 
ralmente) femminile, in quanto descrive, all'interno di un io maschile, lo spazio mentale occupato dal principio del piacere. Il procedere a capo chino (per il peso angoscioso dei pensieri) è il segnale di una soggettività che ha perso, oltre che il contatto con il reale, anche il controllo sulla propria identità, che il desiderio espone al rischio della alienazione: il femminile, infatti, invade la mente con i suoi fantasmi, che si proiettano sul reale, allegorizzandolo. Possiamo allora immaginare che il protagonista della Commedia proceda «a capo chino", nel momento di smarrirsi. La selva oscura è la prima allegoria, intensamente femminile, che il suo desiderio genera.?

Il femminile viene messo in forte rilievo dalla seconda terzina, in cui la caoticità espressiva della selva, per il poeta che la descrive, si manifesta nella contorsione della sintassi ("quanto ... dura») e nell'accumulazione degli aggettivi (4 in un solo verso):

Ahi quanto a dir qual era è cosa dura esta selva selvaggia e aspra e forte che nel pensier rinova la paura!

Qui però al simbolismo generico (che anche i significanti sembrano declinare al femminile come "fonosimbolismo in atto del disordine in cui si trova l'uomo caduto nello stato di peccato"), ${ }^{10}$ si aggiunge un altro più personale piano di significazione, messo in luce dai rinvii intertestuali. Avvertiamo innanzitutto echi delle petrose:

Così nel mio parlar voglio essere aspro

com'è ne li atti questa bella petra,

la quale ognora impetra

maggior durezza...,

che investono la figura della selva dei valori di una femminilità non semplicemente archetipica, ma densamente, angosciosamente autobiografica: la donna come ossessivo oggetto di desiderio e costante principio di scrittura, inflessibile nella sua esigenza di adeguamento della parola al sentimento («Così nel mio parlar voglio esser aspro» = "Ahi quanto a dir qual era è cosa dura»), che ora soltanto, nel quadro di una conclusiva sperimentazione metafisica, trova, nella "selva oscura", il simbolo definitivo. E si avverta poi l'allusione a un testo canonico per la rappresentazione del femminile del medioevo, le parole della sposa nel Cantico dei Cantici $(8,6)$ :

9. Curiosamente, l'immagine del poeta a "capo chino» riappare, ma con diversa intenzione, in Inf., XV, 44, nell'episodio di Brunetto Latini: «Io non osava scender de la strada / per andar par di lui; ma 'l capo chino / tenea com'uom che reverente vada".

10. Cfr. Gianfranco ConTini, La forma di Dante: il primo canto della "Commedia», in Postremi esercizi ed elzeviri, Torino: Einaudi, 1998, p. 67. Con la consueta acutezza, lo studioso osserva nel canto, attraverso il computo delle allitterazioni, una progressione della scrittura dal caos all'ordine. 
Pone me ut signaculum super cor tuum,

Ut signaculum super brachium tuum,

Quia fortis est ut mors dilectio,

Dura sicut infernus aemulatio...

La percezione della donna come soggetto-oggetto di un desiderio il cui eccesso seduce e travia, come spazio di alterità interno alla coscienza, frontiera morale dell'io maschile, votato alla dipendenza e al culto una parte, ma esposto all'alienazione e alla morte dall'altra, pur nell'ideologia arcaica di un inno nuziale, ha nel Cantico vibrazioni di tale intensità, che non sorprende il fascino che esso esercitò sulla spregiudicata metafisica del desiderio degli stilnovisti. ${ }^{11}$ I versi che precedono quelli ora citati ispirano, infatti, l'incipit di un sonetto cavalcantiano (Chi è questa che vèn chogn'om la mira) non estraneo alla ideazione del Proemio: "Quae est ista quae ascendit de deserto, deliciis affluens, innixa super dilectum suum?». ${ }^{12}$

Ma è sul piano autoermeneutico, cioè nella personalissima rete intertestuale attivata dal suo significante, che la selva denuncia il simbolismo femminile che l'ha generata. Si consideri il verso 7: "Tant'è amara che poco è più morte». La distinzione amaroldolce è equiparata a quella, presente fin dal v. 2, oscurolchiaro. Si veda, al riguardo, Conv., IV, ii, 3-4:

Dico adunque che a me conviene lasciare le dolci rime d'amore le quali solieno cercare li miei pensieri; e la cagione assegno, perché dico che ciò non è per intendimento di più non rimare d'amore, ma però che ne la donna mia nuovi sembianti sono appariti li quali m'hanno tolto materia di dire al presente d'amore. Ov'è da sapere che non si dice qui li atti di questa donna essere «disdegnosi e fieri» se non secondo l'apparenza; sì come, nel decimo capitolo del precedente trattato, si può vedere come altra volta dico che l'apparenza de la veritade si discordava. E come ciò può essere, che una medesima cosa sia dolce e paia amara, o vero sia chiara e paia oscura, qui[vi] sufficientemente vedere si può.

Amarezza e oscurità sono qui predicati non del femminile in generale, ma di quel particolare femminile (la «donna gentile» come è descritta nella canzone Le dolci rime d'amor chio solia e nella ballata Voi che savete ragionar d'amore, cui fa riferimento il brano appena citato) che si presenta al poeta con l'apparenza della negatività (il disdegno, la irrazionalità). ${ }^{13}$ Mentre la seconda terzina evo-

11. Sul Cantico e i suoi commenti nella Commedia cfr. Lino PerTILE, La puttana e il gigante. Dal cantico dei cantici al paradiso terrestre, Ravenna: Longo, 1998.

12. Il frammento è citato in Conv. II, 5: «... la sua sposa e secretaria Santa Ecclesia de la quale dice Salomone: "Chi è questa che ascende del diserto, piena di quelle cose che dilettano, appoggiata sopra l'amico suo?"».

13. Bisogna però segnalare (con Anna Maria CHIAVACCI LEONARDI-Inferno, Milano: Mondadori, 1998 [1991], p. 11) anche il paragone biblico "Inveni amariorem morte mulierem» (Eccl. $7,27)$, in cui la donna occupa il luogo logico che, nella Commedia, ha la selva. 
cava, attraverso la durezza e l'asprezza, l'immagine della donna-pietra che nega al poeta la propria sessualità, il verso 7 evoca, attraverso l'amarezza che è sinonimo di oscurità, la donna-filosofia che nega al poeta le sue verità. La selva (oscura, dura, aspra, amara) riassume quella negatività del femminile con cui Dante si è scontrato nelle varie fasi della sua ricerca, e che ha rappresentato per lui ogni volta l'ostacolo espressivo da superare, la barriera intellettuale da infrangere. Il suo significante accumula tali valori, fissandoli in una immagine di materializzata malvagità.

A tale malvagità si oppone, con ulteriore dicotomia, il ben che il poeta dice di avervi trovato (Virgilio, principio maschile di salvezza che positivamente agisce dall'interno della selva), ma che potrà essere descritto (trattato) solo dopo che siano state narrate le altre cose $e^{14}$ che Dante ha visto nella selva (cioè le tre fiere):

ma per trattar del ben ch'i vi trovai, dirò de l'altre cose ch'i' v'ho scorte.

La perplessità dei critici sulla imprecisione dei dettagli spaziali (tanto Virgilio quanto le fiere si trovano in realtà fuori della selva, sull'erta che conduce alla cima del colle) non tiene conto della natura simbolica di queste immagini, che si concatenano secondo una logica più onirica che romanzesca. Fantasma di angosce antiche che claustrofobicamente avviluppano il poeta, la selva contiene anche il suo opposto: essa è il male, ma al suo interno si occulta il bene. ${ }^{15}$ Perché ciò sia narrabile, l'immagine si scompone in dicotomie simboliche che visualizzano (in una manichea psicomachia onirica) la lotta fra le due istanze generiche che scindono l'io e se ne contendono il controllo. La materialità vegetale della selva dà luogo ai tre elementi narrativi delle scene seguenti: e cioè il paesaggio (che si apre come scenario drammatico di una serie di azioni), le fiere (nella cui animalità si trasfigura e pluralizza l'astratta femminilità della selva) e Virgilio (la cui umanità si connota come maschile, poiché si oppone alla animalità delle fiere).

Io non so ben ridir com'i' v'intrai, tant'era pien di sonno a quel punto che la verace via abbandonai.

14. Come ha ben visto Gianfranco CONTINI, l'espressione, lungi dall'essere generica e imprecisa, significa, proprio attraverso l'ellissi, il tabù che grava su oggetti innominabili (La forma di Dante..., p. 75).

15. Il De Vulgari, che utilizza il simbolo della silva per definire la materialità idiomatica dei volgari municipali in opposizione all'ideale volgare illustre che dovrà sublimarli poeticamente in un superiore registro di lingua, presenta un'analoga struttura simbolica: da una parte la silva, ciò̀ $\mathrm{i}$ «saltus et pascua Ytaliae», dall'altra (ma anche al suo interno) la "pantheram... redolentem ubique et necubi apparentem» $(\mathrm{I}, \mathrm{xvi}, 1)$. 
Nella lucida prospettiva a posteriori del poeta che narra, la selva è percepita ormai come esperienza onirica, cioè inesplicabile nelle sue cause. ${ }^{16} \mathrm{Il}$ significante della via viene ripreso, ma con una chiara coscienza della responsabilità individuale del traviamento (al principio era smarrita, qui è l'io che l'ha abbandonata), ${ }^{17}$ e con una importante variante: verace, invece di diritta. Verace fa gruppo con gli altri predicati del maschile, mentre la selva viene implicitamente ridefinita come falsa (ma la dicotomia apparenza / veritade era già presente, come s'è visto, fra i predicati della "donna gentile», e quindi implicita nella alternativa dolce / amara). Il sonno che è causa del traviamento è, certo, il metaforico sonno della ragione, ${ }^{18} \mathrm{ma}$ anche il letterale stato di sonnolenza che espone la mente ai fantasmi dell'immaginazione, che agiscono secondo la logica del sogno, ignara del principio di causalità. In effetti, la comprensione della genesi onirica della selva è necessaria perché siano intese nella loro densità simbolica e nei loro rapporti associativi le apparizioni seguenti.
Ma poi ch'i' fui al piè d'un colle giunto,
là dove terminava quella valle
che m'avea di paura il cor compunto,
guardai in alto e vidi le sue spalle
vestite già de' raggi del pianeta
che mena dritto altrui per ogne calle.

Con procedimento di spostamento proprio del sogno, la selva è diventata un paesaggio dal rilievo disuguale (una valle dominata da un colle). ${ }^{19}$ Viene così neutralizzata l'opposizione "chiuso - aperto", ed attivata quella "basso - alto». La selva, che ora è una valle, acquista un'altra determinazione del femminile (quella del basso, appunto) rispetto ad un elemento alto, il colle, su cui si trasferiscono i valori maschili veicolati prima dal simbolo della via diritta e verace. La luce del sole, che spunta dall'alto del colle, esplicita l'elemento generico maschile che il lettore aveva intuito già nel terzo verso, opponendosi, come luminosa razionalità, alla buia irrazionalità del femminile. Si osservi anche che mentre la «diritta via» si opponeva alla «selva oscura» nella dimen-

16. Analoga genesi onirica ha la selva di Purg., XXXII ("disegnerei com'io m’addormentai...», 68), scenario di simbolismi affini a quelli del Proemio.

17. Rispetto alla iniziale «dispersione del soggetto" (cfr. Gianfranco ConTINI, La forma di Dante..., p. 68), l'io del protagonista va poco a poco conquistando il controllo sull'azione.

18. Molto pertinenti sono, al riguardo, le osservazioni di Anna Maria ChIAVACCI LeONARDI (op. cit., p. 12): «Sul piano filosofico, l'etica aristotelica, ripresa da Tommaso e da Dante, non ammette che l'intelletto possa scegliere deliberatamente il male; esso lo sceglie appunto "per errore", in quanto offuscato, credendolo cioè un bene».

19. Al verso 77 il colle sarà ridefinito "il dilettoso monte», eco del biblico "Quis ascendet in montem Domini», Ps., 23, che ispirerà, in Purg., XXX, 74, il rimprovero di Beatrice a Dante: «Come degnasti d'accedere al monte?». In Inf., XV, 50 la selva viene senz'altro definita «una valle» (e lo spostamento lessicale è certamente mediato dalla espressione biblica «in valle lacrimarum", di Ps., 83, 7). In entrambi i casi le mediazioni scritturali interagiscono con il simbolismo dei generi, potenziandone la forza espressiva. 
sione del tempo (prima si smarrisce la via e poi ci si ritrova nella selva), il colle si oppone alla valle sul piano spaziale (si cerca di andare dal fondo della valle alla sommità del colle, attraverso un "dritto... calle»). L'immagine onirica si sviluppa, così, in un'azione già tendenzialmente romanzesca. ${ }^{20}$
Allor fu la paura un poco queta, che nel lago del cor m'era durata la notte ch'i' passai con tanta pieta.

Notevole è qui la contrazione delle durate: il passaggio dalla oscurità notturna della selva alla luce diurna che viene dal colle si produce come transito da un luogo ad un altro, come se, nello stesso istante, nella selva fosse notte e sul colle giorno. ${ }^{21}$ Si tratterebbe, sull'asse del tempo, di una incongruenza analoga a quella osservata ora sull'asse dello spazio. Ma anche in questo caso è in un senso simbolico, e secondo una logica di tipo onirico, che le immagini hanno senso: la notte, come la paura e la pieta, è attributo femminile della selva, che il protagonista può ora descrivere dall'esterno, e il poeta oggettivare narrativamente, perché la loro prospettiva generica si è spostata, situandosi dalla parte della razionalità ordinatrice maschile. Significativa è poi l'insistenza sulla paura (che appare cinque volte nella scena iniziale: vv. $6,15,19,44,53)$, tema cavalcantiano che esprime l'angoscia del desiderio e gli effetti distruttivi che l'amata produce sulla persona e nella mente dell'amante. Dante, che aveva già assimilato il tema nelle Rime e nella Vita Nuova, lo riutilizza qui senza annullare il senso sessuale che aveva in Guido, poiché è simbolicamente legato al femminile l'elemento umido presente nell'espressione il «lago del cor», ${ }^{22}$ che anticipa, come in una catena di associazioni generate dai significanti, la grande similitudine marina dei versi seguenti, straordinario compendio di valori archetipici:

E come quei che con lena affannata, uscito fuor del pelago a la riva, si volge a l'acqua perigliosa e guata, così l'animo mio, ch'ancor fuggiva, si volse a retro a rimirar lo passo che non lasciò già mai persona viva.

20. Il gesto con cui il protagonista solleva lo sguardo verso l'alto aprendo il suo orizzonte, se da una parte evoca un paesaggio morale di tipo religioso (cfr. Ps., 120, 1: «Levavi oculos meos in montes, unde veniet auxilium mihi»), dall'altra richiama esperimenti narrativi più personali (cfr. Un dì si venne a me, 8: "guardai e vidi Amore, che venia», per il nesso "guardai ... vidi», e poi Vita Nuova, XIV, 4 e XXXV, 2, in cui il sintagma «levai li occhi ... vidi» drammatizza la messa a fuoco visiva, da parte del poeta, di Beatrice e della "donna gentile», rispettivamente).

21. Edoardo SANGUINETI, che mette in rilievo «il sistema compatto di archetipi, di immemorabile spessore simbolico» presente nel canto, parla, a proposito di questi versi, di «rito di passaggio" (Lectura Dantis tenuta nella "Casa di Dante» in Roma il 17 novembre 1996).

22. Nel sonetto dubbio, ma ben dantesco, Nulla mi parve mai, la differenza di temperatura fra il desiderio dell'amata e quello dell'amante viene descritta così: «...'l suo desio nel congelato lago, / ed in foco d'amore il mio si posa». 
Ma di nuovo ai simboli antropologicamente convenzionali Dante aggiunge valori poeticamente autografici. Nel terrore del naufrago che affannosamente sfugge all' «acqua perigliosa» che vorrebbe risucchiarlo, si avverte l'eco degli agghiaccianti paesaggi onirici delle petrose, nei quali la freddezza femminile viene rappresentata come combinazione di durezza e umidità (Amor, tu vedi ben, 25-30):
Segnor, tu sai che per algente freddo
l'acqua diventa cristallina petra
là sotto tramontana ov'è il gran freddo,
e l'aere sempre in elemento freddo
vi si converte, sì che l'acqua è donna
in quella parte per cagion del freddo...

Alle petrose bisogna in effetti riandare per osservare la genesi simbolica ed espressiva della selva proemiale, nella quale il poeta ha raffigurato la propria esperienza del femminile, nel suo estremo polo negativo. Percepiamo meglio, in quest'ottica, l'ineluttabilità della sconfitta morale che essa (la donna-selva) produce nell'io che ne resta irretito. Il verso «che non lasciò giammai persona viva» (in cui il relativo va inteso come soggetto, l'aggettivo come predicato e il sostantivo come indefinito) allude molto più alla malattia mortale del desiderio, secondo medici e poeti, ${ }^{23}$ che non al vizio e al peccato (dai quali l'ani$\mathrm{ma}$, con il pentimento, può sempre evadere). L'inammissibilità teologica, per un cristiano, del concetto che il verso enuncia (il male come condanna irreversibile), scompare se si tiene conto del suo significato sessuale, che allude al dualismo dei generi così come esso viene descritto nella letteratura del desiderio, che lo radicalizza fino a concepirlo come sconfitta mortale dell'io. ${ }^{24}$

Ancora al simbolismo dei generi bisogna ricorrere per chiarire un altro dei punti dubbi del I canto. Posta una relazione maschile fra l'animo e il movimento («l'animo mio ch'ancor fuggiva»), ed una relazione femminile fra il corpo e l'immobilità ("èi posato un poco il corpo lasso»), il verso che conclude la terzina successiva,

Poi ch'èi posato un poco il corpo lasso, ripresi via per la piaggia diserta, sì che 'l piè fermo sempre era 'l più basso,

23. Si pensi a definizioni come queste: «Amore quanto a morte vale a dire... Ahi come, com'è morto bene / qual'ha, sì come me, in podestate (Guittone, Ahi Deo che dolorosa, 28-32)»; «Di sua potenza (di Amore) segue spesso morte, / se forte — la vertù fosse impedita, / la quale aita - la contraria via... non pò dire om ch'aggia vita, ché stabilita — non ha segnoria (Guido Cavalcanti, Donna me prega, 35-41)». Proprio questi ultimi versi risuonano nel Proemio: la lonza «impediva.. il mio cammino» e la lupa «non lascia altrui passar per la sua via / ma tanto lo 'mpedisce che l'uccide».

24. Si tratta, cioè, del «doloroso passo» di Inf., V, 114: il desiderio, privo del «fedele consiglio de la ragione», conduce ineluttabilmente alla morte. 
esprime la tensione del soggetto verso i valori del maschile, che si manifesta nella doppia associazione fra movimento ed altezza, da una parte, e fra staticità e bassezza dall'altro. Sul piano letterale, l'immagine dei piedi alternativamente fermi ed in moto significa il movimento ascensionale ininterrotto, per cui il piede d'appoggio è sempre il più basso e quello in movimento il più alto (se il poeta tornasse indietro, e scendesse invece di salire, sarebbe il contrario). ${ }^{25} \mathrm{Ma}$ la connessione simbolica fra ciò che è fermo e ciò che è in basso, entrambi subordinati a ciò che è in moto e a ciò che è in alto, è plausibile solo sul piano generico del femminile, pulsione negativa dell'io qui contrarrestata dalla opposta pulsione positiva maschile. La dicotomia ideale e archetipica fra principio attivo-maschile (l'animo) e principio passivo-femminile (il corpo) si concreta nella distinzione fra piede in movimento e piede fermo. ${ }^{26}$

Ed ecco, quasi al cominciar de l'erta, una lonza leggera e presta molto, che di pel macolato era coverta; e non mi si partia dinanzi al volto, anzi 'mpediva tanto il mio cammino, ch'i' fui per ritornar più volte vòlto.

Il femminile riappare con forza nelle tre fiere che affrontano il protagonista. La prima rappresenta il desiderio perverso e la seduzione. Leggerezza, agilità, screziatura della pelle ${ }^{27}$ sono i segni della lussuria, descritta con i caratteri dell'oggetto che la suscita in una sensibilità maschile, cioè un corpo femminile che attrae l'attenzione del poeta e ne perverte la volontà. L'apparizione della

25. È questa la lettura più ovvia (per Boccaccio, il poeta «mostra l'usato costume di coloro che salgono, che sempre si ferman più in su quel piè che più basso rimane»).

26. L'immagine del piede dell'anima, con cui la teologia rappresentava la dialettica interiore fra ragione e appetito (per esempio in Agostino: «Il piede dell'anima va inteso rettamente come amore; il quale, quando è vile o indegno, è chiamato cupidigia o lussuria; e quando invece è giustamente orientato, è diletto o carità», Enar. In Psalm. XCIV, I), ha fatto pensare che Dante alluda alla difficoltà di liberarsi dalla concupiscenza, per cui la sua sarebbe un'andatura claudicante: il piede in movimento è il destro, che trascina il sinistro (cfr. John FRECCERO, Dante. La poetica della conversione, Bologna: Il Mulino, 1989, p. 53-90). Sembra però più plausibile che Dante sottolinei qui proprio il contrario, e ciò̀ che sale spedito verso la luce. In effetti si coglie un'allusione (per antitesi) alla meretrix di Proverbia, 1-9: «Ne attendas fallaciae mulieris; favus enim distillans labia meretricis, et nitidius oleo guttur eius... Pedes eius descendunt in mortem et ad inferos gressus illius penetrant. Per semitam vitae non ambulant, vagi sunt gressus eius et investigables»(5). Il simbolo dei piedi della meretrice si figuralizza poi nella «mulier stulta et clamosa» che «sedit in foribus domus suae, super sellam in excelso urbis loco, ut vocaret transeuntes per viam, et pergentes itinere suo"(9), immagine a sua volta adombrata dalla lonza che, in Dante, apparirà subito dopo.

27. Francesca e Paolo colpiscono l'attenzione di Dante perché "paion sì al vento esser leggeri» (V, 75), mentre in Aen., I, 323, la compagna di Venere è «succinctam ... maculosae tegmine lyncis» (cfr. Anna Maria CHIAVACCI LEONARDI, op. cit., p. 17). La rapidità con la quale la seduzione femminile colpisce la mente maschile è tema tipicamente cavalcantiano: (Voi che per li occhi, 9-11) "Questa virtù d'amor che m'ha disfatto / da' vostr'occhi gentil' presta 
lonza interrompe il percorso ascensionale dell'io, e lo fa indietreggiare. Elementi fantastici molto simili li troviamo in un sogno narrato al principio del canto XIX del Purgatorio, in cui Dante confessa come esperienza propria il turbamento della immaginazione prodotto dalla accidia. Il canto precedente si conclude con il poeta che si abbandona alla sonnolenza che l'ha invaso (XVIII, 141-145):
novo pensiero dentro a me si mise, del qual più altri nacquero e diversi;
e tanto d'uno in altro vaneggiai, che li occhi per vaghezza ricopersi, e 'l pensamento in sogno trasmutai.

La evagatio mentis e la somnolentia fanno parte del quadro morale della accidia. ${ }^{28}$ Il sogno del poeta (una femmina balba che lo sguardo concupiscente trasforma in seducente sirena) esemplifica il traviamento morale che la donna produce quando è oggetto di desiderio per una immaginazione maschile non orientata dalla ragione (quindi sonnolenta e delirante "per vaghezza»). Si osservi il traviamento di Ulisse, "del suo cammin vago", che la sirena orgogliosamente vanta (analogo a quello di Dante, finché Virgilio non lo sveglia e lo obbliga a riprendere il cammino):

mi venne in sogno una femmina balba, negli occhi guercia, e sovra i piè distorta, con le man monche, e di colore scialba.

Io la mirava; e come 'l sol conforta le fredde membra che la notte aggrava, così lo sguardo mio le facea scorta la lingua, e poscia tutta la drizzava in poco d'ora, e lo smarrito volto, com'amor vuol, così le colorava. Poi ch'ella avea 'l parlar così disciolto, cominciava a cantar sì, che con pena da lei avrei mio intento disciolto. «Io son» cantava «io son dolce serena, che' marinari in mezzo mar dismago; tanto son di piacere a sentir piena!

si mosse: / un dardo mi gittò dentro dal fianco»; (Era in penser d'amor, 31-36) «i' dissi: 'E' mi ricorda che ' $\mathrm{n}$ Tolosa / donna m'apparve accordellata istretta, / amor la qual chiamava la Mandetta; / giunse sì presta e forte, / che fin dentro, a la morte, / mi colpîr gli occhi suoi'”. 28. Sull'accidia e sulla complessità della disposizione morale che il termine indica, è necessario ricorrere a Tommaso D'AQUINO $\left(S . T ., 2^{\mathrm{a}}-^{2}\right.$, 35, 4), che, sintetizzando la riflessione teologica sul tema, distingue nel complesso tristitia-acedia tali componenti: «desperatio, pusillanimitas, torpor circa praecepta, otiositas, somnolentia, rancor, amaritudo, malitia, evagatio mentis circa illicita, importunitas mentis, curiositas, verbositas, inquietudo corporis, instabilitas». 
Io volsi Ulisse del suo cammin vago

al canto mio; e qual meco si ausa, rado sen parte; sì tutto l'appago...

Le coincidenze fra la femmina balba e la lonza sono notevoli. Entrambe hanno su Dante un effetto ipnotico, che l'episodio del Purgatorio presenta come conseguenza della accidiosa somnolentia alla quale il poeta cede, e quello del Proemio come il potere di seduzione che un corpo animalescamente femminile esercita su un io "pien di sonno». Lo sguardo obnubilato dell'accidioso è comparato con i raggi del sole, mentre l'anatomia della femmina balba è paragonata alle "fredde membra che la notte aggrava»; il che mostra che qui è operante la stessa simbologia generica del Prologo, poiché il maschile è associato al caldo, alla luce, al giorno, allo spirito, mentre il femminile è associato al freddo, al buio, alla notte, al corpo. Ma soprattutto è significativa la funzione di traviamento che hanno le due figure femminili: come la lonza fa indietreggiare Dante («fui per ritornar più volte volto»), così la sirena «volse Ulisse». 29 Il movimento orientato e rettilineo di entrambi è impedito da figure femminili di seduzione che la ragione smaschera come prodotti della immaginazione maschile pervertita dal desiderio.

L'analogia fra la lonza e la "femmina balba» aggiunge un elemento alla identificazione ideale di Dante con Ulisse (come si sa, uno dei grandi motivi della Commedia), simili non solo per la concezione eroica dell'esistenza come viaggio di conoscenza al di là dei limiti imposti alla condizione umana, ma anche per la comune esperienza del femminile come impedimento e interruzione del cammino: entrambi devono evitare, con stratagemmi umani o con l'aiuto divino, le trappole che la seduzione femminile tende loro durante il viaggio.
Temp'era dal principio del mattino, e '1 sol montava 'n sú con quelle stelle ch'eran con lui quando l'amor divino mosse di prima quelle cose belle; sì ch'a bene sperar m'era cagione di quella fiera a la gaetta pelle l'ora del tempo e la dolce stagione;

29. Funzione analoga ha la Circe che «ritenne più d'un anno» Ulisse (Inf., XXVI), e fra gli ammonimenti di Beatrice a Dante (Purg., XXXI), c'è anche quello che «altra volta, udendo le serene, sie più forte» (44-45). Non mi sembra pertinente, riguardo all'espressione "volsi Ulisse», l'osservazione che in Omero l'eroe sfugge alle sirene. Ciò che Dante sottolinea, delle sirene, è il loro potere di distrazione, cioè il fascino perverso che esse esercitarono perfino su Ulisse, così vago del suo cammino. Il verbo che Dante usa, sia qui che nel Proemio, volgere, significa letteralmente "girarsi», cioè distrarre l'attenzione e la volontà dalla meta del cammino (e in un senso analogo è usato, ancora nel Proemio, v. 88: "Vedi la bestia per cu' io mi volsi», poi in Inf., II, 62-63: «ne la diserta piaggia è impedito / sì nel cammin, che volt’e per paura", in Purg., XXX, 130: «e volse i passi suoi per via non vera» e in Purg., XXXI, 35-36: «Le presenti cose / col falso lor piacer volser miei passi»). 
Trasparenti sono i simboli del maschile che neutralizzano il potere di seduzione della lonza: la luce diurna del mattino; il sole che comincia il suo movimento ascensionale; la costellazione dell'ariete, che segna l'inizio della primavera ed è quindi associata all'azione fecondante del sole; l'atto creatore dell' «amor divino" che imprime il movimento all'universo. Il sintagma "quelle cose belle» è poi in significativa antitesi con le «altre cose» del v. 9: se queste alludono alla oscenità del caos, quelle indicano, invece, la bellezza del cosmo, il che ci riporta alla dicotomia originaria delle cosmologie platonica e manichea. Il principio maschile dell'ordine sembra imporsi, quindi, su quello femminile del disordine.

Il femminile riprende il sopravvento con la seconda fiera:

ma non sì che paura non mi desse

la vista che m'apparve d'un leone.

Questi parea che contra me venisse

con la test'alta e con rabbiosa fame,

sì che parea che l'aere ne tremesse.

Accusata è la presenza di stilemi cavalcantiani, in particolare di un sonetto per più motivi legato al Proemio. Si tratta di Chi è questa che vèn, il cui secondo verso recita: "che fa tremar di chiaritate l'âre». ${ }^{30}$ Sono genericamente cavalcantiani anche l'ostilità belligerante che il fantasma manifesta nei confronti dell'io, e la conseguente paura del poeta. Nella prima quartina di un altro sonetto, Io temo che la mia disaventura, troviamo alcuni degli elementi espressivi e tematici del I canto dell'Inferno (sostanzialmente, quelli generati dalla paura):

Io temo che la mia disaventura non faccia sì ch'io dica: «I' mi dispero», però ch'i'sento nel cor un pensero che fa tremar la mente di paura. ${ }^{31}$

Lo stesso vale per il congedo di Io non pensava (47-53):

e prego umilemente a lei tu guidi li spiriti fuggiti del mio core, che per soverchio de lo su' valore eran distrutti, se non fosser vòlti,

30. Sulle fonti scientifiche dell'immagine, e sul fenomeno della «scintillazione», cfr. Domenico De Robertis (a cura di), G. C., Rime, Torino: Einaudi, 1986, p. 17.

31. Questo sonetto rinvia a sua volta a quello di Guittone S'el si lamenta (la cui prima quartina è citata dai versi 55 e sgg. del Proemio): «S'el si lamenta null'om di ventura, / a gran ragion mi movo a lamentanza, / sì come om, che si credia in altura, / ed è caduto e tornato in bassanza. / E vo piangendo e moro di paura, / poi che mi vidi in tanta sicuranza / di quella, ch’è più bella criatura / che Deo formasse senza dubitanza». 
e vanno soli, senza compagnia,

e son pien' di paura.

Però li mena per fidata via... 32

È chiaro, però, che l'immagine che ha guidato Dante nella rappresentazione del leone è quella della donna descritta da Guido in Chi è questa che vèn, la quale a sua volta era stata modellata, come si è visto, sulla sposa del Cantico («terribilis ut castrorum acies ordinata», 6, 3). Nel suo approfondimento parallelo delle componenti visionarie e distruttive del desiderio, Guido aveva trovato nel testo biblico lo straordinario esempio di un femminile che seduce ed atterrisce nello stesso tempo. La sua parodia del testo sacro fa del desiderio l'unica dimensione tragica dell'esistenza, subordinando paradossalmente alla sua logica sessuale ogni contenuto culturale (religione, filosofia, letteratura...). Soprattutto dipende dal desiderio (cioè dalla conoscenza del femminile) la salvezza esistenziale e metafisica del soggetto maschile: «non fu sì alta già la mente nostra / e non si pose 'n noi tanta salute, / che propiamente n'aviàn canoscenza" (Chi è questa che vèn, 12-14). Dal canto suo Dante, dopo aver esposto nella lonza l'aspetto sirenico del femminile, riprende pari pari da Guido ed attribuisce ad un superbo e famelico leone quegli effetti di metafisico tremore che nell'amico suscitava l'amata. Mentre la lonza rappresenta il lato seduttivo della femminilità, il leone ne rappresenta il lato biblicamente punitivo. Il vizio della superbia, cui il leone sembra alludere, non solo non contraddice il suo simbolismo generico, ma anzi ribadisce una connessione concettuale del tutto normale in Dante. ${ }^{33}$

Con ulteriore associazione onirica (che l'anacoluto «una lupa ... questa» sembra voler riflettere nella struttura grammaticale), la «rabbiosa fame» del leone genera la magrezza della lupa:

Ed una lupa, che di tutte brame sembiava carca ne la sua magrezza, e molte genti fé già viver grame, questa mi porse tanto di gravezza con la paura ch'uscia di sua vista, ch'io perdei la speranza de l'altezza.

Figura riassuntiva delle due fiere precedenti, la lupa ne incrementa i valori negativi, declinando al femminile un vizio, la avarizia, che solo in quanto vincolata ad attività mercantili normalmente maschili può essere considerata come la suprema perversione della società civile (gli esempi di avari, sia nel-

32. Il verso «e vanno soli senza compagnia» appare quasi intatto all'inizio di Inf., XXIII: «Taciti, soli, sanza compagnia».

33. Superba è la "donna gentile» in Conv., III, ix, 1: "parendo a me questa donna fatta contra me fiera e superba alquanto", e Beatrice in Purg., XXX, 79-80: "Così la madre al figlio par superba, / com'ella parve a me...". 
l'Inferno che nel Purgatorio, sono di ecclesiastici). ${ }^{34}$ In Convivio, IV, 3, l'avarizia è antitetica al diritto appetito e alla vera conoscenza che inducono l'uomo a sfuggire le ricchezze, quando non sono «ordinate... ad alcuno necessario servizio». E i due elementi che in prima istanza caratterizzano la lupa sono la famelicità (le brame di cui è segno la magrezza) e il potere economicamente distruttivo (per lei molte genti vivono grame). Lidea di avarizia associabile ad essi è molto ampia, poiché include da una parte la febbrile accumulazione di ricchezza, dall'altra gli effetti depauperanti (sugli altri) che essa genera. Si tratterebbe insomma di un capitalismo selvaggio basato sullo sfruttamento e l'usura (criticato con durezza dai movimenti pauperisti dell'epoca).

Ma l'idea concomitante che fa dell'avarizia un vizio simbolicamente femminile (quindi lupa e non lupo) è la prostituzione. Il principale nesso intertestuale è il sonetto 167 del Fiore, nel quale la seduzione femminile a scopo di lucro è associata esplicitamente al comportamento della lupa:
La lupa intendo che, per non fallire a prender ella pecora o montone, quand'e' le par di mangiar [i]stagione, ne va, per una, un cento e più asalire. Così si dé la femina civire sed ella avesse in sé nulla ragione: contra ciascuno riz[z]ar dé il pennone per fargli nella sua rete fedire...

La connessione lupa-prostituta è certo un luogo comune della cultura popolare, che spiega perfettamente l'uso ampio che Dante ne fa nel Proemio. Nella Cronica di Compagni troviamo però due luoghi che potrebbero aver suggerito al poeta l'idea di una lupa che impedisce o no il passaggio («non lascia altrui passar per la sua via», spiegherà poco dopo Virgilio):

2, 28: I Ghibellini e Bianchi, che erano rifuggiti in Siena, non si fidavano starvi, per una profezia che dicea: «La lupa puttaneggia», ciò è Siena, che è posta per la lupa; la quale quando dava il passo, e quando il toglieva...

2, 36: I Bianchi e i Ghibellini di Firenze... cavalcarono ad Arezo con soldati Pisani. I Sanesi dierono loro il passo: perché i cittadini di Siena marcavano bene con anbo le parti; e quando sentivano i Bianchi forti, li sbandiano, ma il bando era viziato, che non agravava: davano aiuto a' Neri nelle cavalcare, e mostravansi fratelli: e però parlò di loro una profezia, la quale fra l'altre parole della guerra di Toscana dicea: «La lupa puttaneggia»; che per la lupa s'intende Siena...

34. La comparazione con il passo scritturale normalmente addotto come fonte per le tre fiere (Geremia, 5, 6: "percussit eos leo de silva, lupus ad vesperam vastavit eos; pardus vigilans super civitates eorum»), mette semmai in rilievo la variante, e cioè quel femminile lupa che, in sé, non ha nulla a che vedere con l'avidità. 
Ma il nesso intertestuale più pertinente, ed anzi decisivo, come si vedrà subito, nella configurazione dell'intero canto, è quello di Convivio, I, 9, in cui l'equivalenza di avarizia e prostituzione tocca il centro della ideologia letteraria di Dante:

Non avrebbe lo latino servito a molti: ché se noi reducemo a memoria quello che di sovra è ragionato, li litterati fuori di lingua italica non averebbono potuto avere questo servigio, e quelli di questa lingua, se noi volemo bene vedere chi sono, troveremo che de' mille l'uno ragionevolmente non sarebbe stato servito; però che non l'averebbero ricevuto, tanto sono pronti ad avarizia che da ogni nobilitade d'animo li rimuove, la quale massimamente desidera questo cibo. E a vituperio di loro dico che non si deono chiamare litterati, però che non acquistano la lettera per lo suo uso, ma in quanto per quella guadagnano denari o dignitate... ma lo volgare servirà veramente a molti. Ché la bontà de l'animo, la quale questo servigio attende, è in coloro che per malvagia disusanza del mondo hanno lasciata la letteratura a coloro che l'hanno fatta di donna meretrice; e questi nobili sono principi, baroni, cavalieri, e molt'altra nobile gente, non solamente maschi ma femmine, che sono molti e molte in questa lingua, volgari e non litterati.

Il primato negativo della avarizia-lupa, così estraneo ad una impostazione teologica convenzionale, che vorrebbe la superbia o l'invidia come vizi principali (e Dante stesso ammette qui, v. 111, che «invidia prima dipartilla»), si deve alla polemica anticlericale che accompagna la riflessione del poeta in ogni fase del suo svolgimento: la prostituzione della letteratura praticata dai chierici è la causa sociale della perversione della cultura, che trasforma il mondo in una selva in cui chiunque è destinato a perdersi, poiché è venuto meno il fondamento della civiltà (la lettera, appunto). ${ }^{35}$ La redenzione civile del mondo dipende dalla creazione di forme alternative di produzione letteraria (nella lingua, nei contenuti, nella identità di produttori e consumatori) che rispondano al bisogno di cultura che una nobiltà nuova, nella mentalità e nei valori, attende. Si osservi la «malvagia disusanza del mondo»: la responsabilità della barbarie non è esclusiva dei chierici; essa è anche di quegli aristocratici di tipo tradizionale che hanno confuso la nobiltà con la nascita ed il patrimonio. Altra e ben diversa è la nuova nobiltà «la quale questo servigio attende».

Proprio la «bontà de l'animo» del pubblico a cui Dante pensa richiama in causa il simbolismo legato ai generi sessuali. Tanto la chiesa quanto l'aristocrazia, infatti, cioè tanto gli oratores quanto i bellatores, sono, per definizione, maschi, essendo inibite alle donne le pratiche relative ai due ordini, quella del

35. Il progressismo illuminato della difesa dantesca del volgare appare ancora più chiaramente se lo si compara con i pregiudizi antivolgari del Novellino, ben più rappresentativo della cultura contemporanea al poeta (cfr., in particolare, LXXVIII, in cui al filosofo che volgarizza la scienza "venne in visione che le dee della scienzia, a guisa di belle donne, stavano al bordello»; e ne danno la colpa a lui perché «volgarizzar la scienzia si era menomar la deitade»). 
sacro e quella della guerra. L' «altra nobile gente», invece, è formata da esseri umani «non solamente maschi ma femmine». Il simbolismo femminile convenzionale ed antico della lupa-prostituta lascia intravedere già un diverso simbolismo, originale e moderno, in cui al femminile dovrà essere almeno parzialmente ascritta quella nuova nobiltà che potrà prosperare quando la lupa sarà ricacciata nell'inferno.

E qual è quei che volontieri acquista, e giugne 'l tempo che perder lo face, che ' $n$ tutti suoi pensier piange e s'attrista; tal mi fece la bestia sanza pace, che, venendomi 'ncontro, a poco a poco mi ripigneva là dove ' 1 sol tace.

La seconda comparazione del Poema, suggerita come la prima dalle associazioni simboliche (lì il lago del cor che generava l'acqua perigliosa; qui le brame di ricchezza che generano l'acquisto e la perdita), presenta un percorso morale inverso, dal positivo al negativo. La lupa determina, infatti, l'indietreggiamento del poeta verso il basso, cioè verso la selva, e verso gli stilemi petrosi ad essa collegati (Io son venuto, 22: «onde l'aere s'attrista tutto e piagne»). La sua oscurità viene però indicata, ora, con un nuovo significante. Il sole che tace, cioè non risplende, implica infatti la doppia analogia luce $=$ parola $/$ buio $=$ silenzio, per la quale l'oscurità della selva si carica di un senso metaforico inedito nella simbologia sessuale antica, e che allude palesemente al potere illuminante del Verbo. ${ }^{36}$ Di questa connessione fra luce e parola Dante si era già appropriato in un altro contesto, ridefinendola in un senso radicalmente moderno. Si è appena visto che la lupa-prostituta allude ad una perversione che nel suo significato più profondo è letteraria. Proprio per questo la selva oscura verso la quale la bestia ripigneva il poeta deve essere pensata, ora, come un luogo letterariamente muto, in cui ciò che non risplende è il sole della letteratura. Il nesso analogico sole-letteratura (volgare) è profeticamente fissato nel paragrafo finale dell'ultimo capitolo del I trattato del Convivio:

... puotesi vedere questo pane, col quale si deono mangiare le infrascritte canzoni, essere sufficientemente purgato da le macule e da l'essere di biado; per che tempo è d'intendere a ministrare le vivande. Questo sarà luce nuova, sole nuovo, lo quale surgerà là dove l'usato tramonterà, e darà lume a coloro che sono in tenebre e in oscuritade per lo usato sole che a loro non luce.

La selva nella quale il poeta sta per essere ricacciato dalla lupa, senza perdere il suo simbolismo femminile originario (il basso loco), acquista un nuovo

36. Il senso illuminativo dell'espressione è colto da John FrECCERO, nel quadro della sua lettura agostiniana del Proemio, in Dante. La poetica..., p. 30. 
senso, molto più problematico nella prospettiva dei generi sessuali: quello delle tenebre prodotte dal silenzio della poesia per la avidità dei litterati.

Mentre ch'i rovinava in basso loco, dinanzi a li occhi mi si fu offerto chi per lungo silenzio parea fioco.

Virgilio è portatore di un simbolismo maschile innanzitutto perché si oppone alle fiere ed impedisce il rovinare di Dante in basso loco, ma anche perché rappresenta una razionalità di tipo poetico la cui funzione è quella di illuminare culturalmente gli esseri umani. Razionalità che certamente non è in contraddizione con i valori cristiani, ma che altrettanto certamente ne ha preso il posto, sussumendoli poeticamente. Ad una letteratura rinnovata dalla poesia in volgare Dante infatti attribuisce, nel Convivio, nel De Vulgari e soprattutto nella Commedia, quel potere illuminativo che la religione attribuisce al messaggio di Cristo. Si tratta di una poesia che, per assolvere questo compito, deve andare ben al di là del suo ambito estrinsecamente retorico, ed illuminare le coscienze con un messaggio di verità umana e divina. Tale funzione la poesia l'ha già avuta in età classica, secondo Dante, con Virgilio, appunto (in base alla visione medievale del Virgilio cristiano). L'immagine che usa Stazio, in Purg. XXII, 67-69, per indicare la consonanza fra la poesia virgiliana e la predicazione evangelica è appunto quella del lampadoforo, cioè di colui che illumina (pur restando soggettivamente escluso dal messaggio di verità che diffonde):

Facesti come quei che va di notte,

che porta il lume dietro e sé non giova,

ma dopo sé fa le persone dotte...

La decadenza della letteratura si produce successivamente (a partire dalla donazione di Costantino), quando l'avidità dei chierici ne perverte i contenuti e la funzione. Il lungo silenzio di Virgilio è dunque il silenzio della poesia, le cui ragioni si sono da tempo offuscate poiché la letteratura è stata trasformata dai litterati, da donna che era, in meretrice. ${ }^{37}$ Inascoltata, la sua parola si è affievolita, ha perso il suo potere illuminante (fioca può solo avere valore visivo, data la simbologia sottesa al brano), che Dante riscopre ora in extremis, un attimo prima di ripiombare definitivamente nel buio irrazionale del silenzio poetico.

Lidentificazione della luce con la parola poetica e di questa con la razionalità (filosofica e religiosa) è forse la struttura simbolica più generale e produttiva della Commedia, quella che ne giustifica globalmente l'ideazione, ed è quindi quella più intimamente legata alla autobiografia intellettuale del poeta.

37. Per un riepilogo della discussione sul significato del «lungo silenzio» di Virgilio, cfr. Gino CASAGRANDE, Parole di Dante. Il «lungo silenzio» di «Inferno", I, 63, in "Giornale storico della letteratura italiana», CLXXIV, 1997, p. 243-254. 
Ed è poi anche il messaggio del Poema che più chiaramente si apre verso i valori della modernità, poiché subordina al linguaggio inteso come poesia, e quindi umanizza, ogni forma di civiltà ed ogni esperienza di cultura (prima fra tutte la religione, che solo attraverso la mediazione trascendentale della poesia conserva modernamente un senso). Il diserto che ora è diventato la selva in cui Dante rischia di perdersi di nuovo, è la barbarie di un mondo privo di cultura, perché privo di poesia. ${ }^{38}$ Per la logica onirica che governa il canto, il personaggio e l'apparizione di Virgilio sembrano generati dal nuovo significato letterario secondo cui la selva è stata simbolicamente ridefinita. Il disperato grido di Dante è la richiesta d'aiuto di un poeta che angosciosamente percepisce lo svuotamento poetico del mondo. Essa è formulata in latino, che è ancora la lingua della cultura, ed afferisce a una dimensione morale in cui poesia e vita, cioè civiltà ed esistenza, sono la stessa cosa. Virgilio è la voce poetica più autorevele, nella tradizione prossima a Dante, e quindi l'unica che può eventualmente ristabilire le ragioni della poesia, salvando Dante e tutti quelli che, come Dante, avvertono l'esigenza di un nuovo sistema di valori che li redima dal diserto a cui la lupa, cioè i litterati, li hanno condannati:

Quando vidi costui nel gran diserto,

«Miserere di me», gridai a lui,

«qual che tu sii, od ombra od omo certo!».

La seconda frase di Dante, e l'alternativa con cui interroga la nuova apparizione, è figura del bivio in cui il poeta si trova, poiché dalla risposta dipenderà il percorso di redenzione che gli si apre. "Od ombra od omo certo" significa infatti: «il nuovo cammino di salvezza, quindi la nuova poesia, che stanno per essere indicati a Dante, implicano un itinerario fisico (omo certo) o metafisico (ombra) ?» L'ambiguità ontologica del personaggio appena apparso prepara il protagonista (ed il lettore) alla zona d'ombra nella quale la poesia dovrà penetrare per compiere la sua funzione redentiva. Virgilio scioglie subito il dubbio, dichiarando umbratile la sua sostanza, e, definendosi poeta, delimita il terreno, poetico appunto, in cui la sua presenza ha senso nel Poema: ${ }^{39}$

Rispuosemi: «Non omo, omo già fui, e li parenti miei furon lombardi,

38. Sulla identificazione di selva e deserto (intesi entrambi come barbarie), cfr. Jacques LE GoFF, Il deserto-foresta nell'Occidente medievale, in Il meraviglioso e il quotidiano nell'occidente medievale, Roma-Bari: Laterza, 1988, p. 25-50.

39. Che Virgilio rappresenti innanzitutto la poesia, è ribadito in Inf., II, 67, quando Beatrice lo prega di soccorrere Dante "con la sua parola ornata», e poi in Inf., II, 113-114, quando Beatrice spiega di averlo scelto come aiutante «fidandomi del tuo parlare onesto / che onora te e quei che udito l'hanno». La funzione razionalizzatrice di cui Virgilio è portatore (che la tradizione esegetica ha indebitamente assolutizzato) è senz'altro subordinata alla sua funzione poetica: è in quanto poeta (non corrotto dall'avarizia) che egli salverà Dante e quanti credono nella necessità di una riforma morale e politica dell'umanità. 
mantoani per patria ambedui.

Nacqui sub Iulio, ancor che fosse tardi, e vissi a Roma sotto '1 buono Augusto nel tempo de li dèi falsi e bugiardi.

Poeta fui, e cantai di quel giusto figliuol d'Anchise che venne di Troia, poi che 'l superbo Ilión fu combusto. $\mathrm{Ma}$ tu perché ritorni a tanta noia? perché non sali il dilettoso monte ch'è principio e cagion di tutta gioia?». "Or se' tu quel Virgilio e quella fonte che spandi di parlar sì largo fiume?», rispuos'io lui con vergognosa fronte. "O de li altri poeti onore e lume, vagliami 'l lungo studio e 'l grande amore che m'ha fatto cercar lo tuo volume.

Tu se' lo mio maestro e ' 1 mio autore, tu se' solo colui da cu' io tolsi lo bello stilo che m'ha fatto onore. Vedi la bestia per cu' io mi volsi: aiutami da lei, famoso saggio, ch'ella mi fa tremar le vene e i polsi».

Il primo dialogo fra Dante e Virgilio è il reciproco riconoscimento di due poeti. Tutti i nessi culturali che vengono evocati, innanzitutto quello fra antichità e modernità, poi quello fra letteratura e società, e infine quello fra vita e morte, cioè fra realtà fisica (Dante) e realtà metafisica (Virgilio), sono filtrati dalla identità poetica che accomuna i due personaggi, da maestro ad alunno, da modello ad imitatore. Se la scena del Proemio ha un significato iniziatico, si tratta della iniziazione di un poeta moderno, ad opera del più prestigioso dei poeti antichi. La strana osservazione "(nacqui sub Iulio, ) ancor che fosse tardi», significa con ogni probabilità: «nacqui troppo tardi per illuminare Cesare con la mia poesia (ed impedirgli di distruggere con la guerra civile la libertà dei Romani e di imporre loro la sua signoria- secondo l'accusa che in varie occasioni Dante insinua contro Cesare).» ${ }^{40}$ L'osservazione seguente, «vissi sotto il buono Augusto", significa al contrario: «nonostante "gli dei falsi e bugiardi" potetti ispirare Augusto con "la divina fiamma" della mia poesia, "onde sono allumati più di mille" (Purg., XXI, 95-96)». Virgilio si presenta non semplicemente come poeta, ma come poeta capace di educare la società romana (attraverso l'imperatore) con il messaggio della sua opera, il cui contenuto epico, il viaggio del "giusto / figliuol d'Anchise», è elemento del piano provvidenziale di redenzione dell'umanità. Egli incarna, quindi, esemplarmente quella funzione che Dante ha riconosciuto nella poesia attraverso tutta la sua riflessione anteriore, funzione civilizzatrice di orientamento culturale e politico della società. 
$\grave{\mathrm{E}}$ in tale dimensione politico-trascendentale della poesia che vengono ripresi, e confermati nei loro valori, i simboli precedenti. Virgilio addita il luogo maschile al quale Dante dovrebbe tendere (il «dilettoso monte»), invece di tornare a "tanta noia»; Dante indica l'elemento femminile (la bestia) che lo ha fatto indietreggiare, e gli chiede aiuto per affrontarla, poiché è sul piano della poesia che può essere risolto il conflitto che la lupa ha scatenato nella sua mente. La conclusione di Virgilio è che Dante dovrà tenere un itinerario differente («altro viaggio»), poiché l'ostacolo rappresentato dalla lupa è insormontabile sul piano della realtà fisica convenzionale. ${ }^{41}$

Ciò che subito sorprende, del discorso di Virgilio sulla lupa, è l'intensificazione dei simboli sessuali con cui la descrive. Il veltro, invece, rappresenta l'inversione di segno e di genere (cioè dal negativo al positivo, e dal femminile al maschile) della lupa.

"A te convien tenere altro viaggio", rispuose poi che lagrimar mi vide, «se vuo' campar d'esto loco selvaggio: ché questa bestia, per la qual tu gride, non lascia altrui passar per la sua via, ma tanto lo 'mpedisce che l'uccide;

Posta una connessione sostanziale fra i due simboli, la selva («esto loco selvaggio») e la lupa ("questa bestia»), questa è ineluttabile principio di morte, come già in precedenza la selva («non lasciò giammai persona viva»). ${ }^{42} \grave{\mathrm{E}}$ di nuovo sul piano simbolico del femminile che viene svolto il tema degli effetti letali del male, un femminile esplicitamente accusato nei suoi caratteri generici (cioè la prostituzione: «Molti son li animali a cui s'ammoglia»):

e ha natura sì malvagia e ria, che mai non empie la bramosa voglia, e dopo 'l pasto ha più fame che pria. Molti son li animali a cui s'ammoglia, e più saranno ancora, infin che 'l veltro verrà, che la farà morir con doglia.

41. Si osservi poi che il verso «mi fa tremar le vene e i polsi» deriva direttamente dal repertorio della sintomatologia erotica ben familiare a Dante: in Vita Nuova, II, 4, all'apparizione di Beatrice, "lo spirito de la vita, lo quale dimora ne la secretissima camera de lo cuore, cominciò a tremare sì fortemente, che apparia ne li minimi polsi orribilmente...». La lupa è dunque, come la selva, compendio di tutte le immagini dolorose di femminilità che hanno costellato la biografia letteraria dell'autore.

42. Come si è già osservato (n. 23), l'effetto distruttivo della lupa è analogo a quello del desiderio secondo Cavalcanti in Donna me prega, 35-37: «Di sua potenza segue spesso morte, / se forte - la vertù fosse impedita / la quale aita - la contraria via» (e il "loco selvaggio» in cui la bestia imprigiona il poeta echeggia le "non già selvagge ... bieltà» che "son dardo", del verso 60 della canzone): l'elemento che Dante raccoglie dall'amico è l'idea del bivio morale, cioè l'alternativa fra vita e morte, che il desiderio impone all'io. 
Questi non ciberà terra né peltro, ma sapïenza, amore e virtute, e sua nazion sarà tra feltro e feltro. Di quella umile Italia fia salute per cui morì la vergine Cammilla, Eurialo e Turno e Niso di ferute. Questi la caccerà per ogne villa, fin che l'avrà rimessa ne lo 'nferno, là onde 'nvidia prima dipartilla.

Il veltro, simbolo riassuntivo dei valori maschili come la lupa lo è di quelli femminili, è descritto con caratteri opposti a quelli della sua antagonista: ${ }^{43}$ mentre questa uccide gli uomini, egli ucciderà lei; mentre la lupa è famelica (di beni e ricchezze), egli si ciberà di cultura (dobbiamo quindi immaginare un tipo di intellettuale che incarni valori opposti a quelli dei chierici), e mentre lei genera nella cupidigia, ${ }^{44}$ egli nascerà nella povertà (il contesto sembra indicare che il feltro è il panno ruvido di case modeste, evocato in antitesi al lusso dei ricchi). ${ }^{45}$

Si osservi ora con attenzione il verso «ma sapienza, amore e virtute», poiché ci permetterà di definire meglio il profilo intellettuale del veltro. La risonanza teologico-trinitaria (analoga a quella che si avverte in Inf., III, 5-6: «fecemi la divina podestate, / la somma sapïenza e 'l primo amore») disturba la percezione di un nesso intertestuale molto più pertinente, ed interno, alla riflessione letteraria di Dante. I "tria magnalia» che in De Vulgari, II, ii, 7 vengono indicati come temi supremi della poesia in volgare, sono la cosiddetta Rota Virgilii: salus (cioè «armorum probitas»), venus (cioè «amoris accensio»), virtus (cioè «directio voluntatis»). Poiché il veltro ciberà il secondo e il terzo dei contenuti della poesia illustre, la sua fisionomia intellettuale si avvicina molto a quella di un poeta. L'elemento mancante, per giunta, la «armorum probitas», è appunto quello che il De Vulgari indica come assente nella poesia italiana («Arma vero nullum latium adhuc invenio poetasse»), ed è sostituito da un altro, la sapienza, che allude inequivocabilmente alla filosofia (Conv., III, xi, 5):

43. La natura «dialettica» del veltro, cioè la sua caratterizzazione per antitesi rispetto alla lupa, indipendente quindi da simbolismi o allegorie predeterminati, fu evidenziata da Antonino PAgliaro (Ulisse. Ricerche semantiche..., p. 494). Già Boccaccio, però, aveva osservato che il poeta "metaphorice chiama "veltro", per ciò che i suoi effetti saranno del tutto contrari all'avarizia come il veltro di sua natura è contrario al lupo» (si noti, però, la neutralizzazione della opposizione di genere).

44. Cfr. Ep., XI, 14: «Cupiditatem unusquisque sibi duxit in uxorem» (Anna Maria CHIAVACCI LEONARDI, op. cit., p. 29).

45. Molto suggestiva è però l'ipotesi avanzata da Paolo BALDAN (Per un veltro dal substrato materico-Inferno, $I$, in "Italianistica», XXI, 2-3, 1992, p. 297-314), che l'espressione "tra feltro e feltro" alluda alla feltratura della carta, il nuovo materiale scrittorio che, per il suo basso costo di produzione, sostituisce la pergamena a partire dalla seconda metà del XIII secolo. L'umiltà laica della carta (il veltro) si contrapporrebbe alla arroganza clericale della pergamena (la $\operatorname{lupa}$ ), come supporto materiale della rivoluzione culturale auspicata dal poeta. 
Pitagora, domandato se egli si riputava sapiente, negò a sé questo vocabulo e disse sé essere non sapiente, ma amatore di sapienza. E quinci nacque poi, ciascuno studioso in sapienza che fosse «amatore di sapienza» chiamato, cioè «filosofo»; che tanto vale in greco "philos» com'è a dire «amore» in latino, e quindi dicemo noi: «philos» quasi amore, e «soph[os]» quasi sapien[te]. Per che vedere si può che questi due vocaboli fanno questo nome di «filosofo», che tanto vale a dire quanto «amatore di sapienza». ${ }^{46}$

Ma proprio nell'ambito della filosofia si è prodotta la rivoluzionaria svolta culturale con la quale Dante e i suoi amici hanno trasformato il linguaggio della poesia (si ricordino, in particolare, il senno bolognese che Bonagiunta da Lucca rimprovera a Guinizzelli in Vo' ch'avete mutata la mainera, e l' "uso moderno" di cui il primo Guido è antesignano secondo Purg., XXVI, 113). Sono indizi sufficienti per concludere che nel veltro Virgilio sta descrivendo un poeta italiano non ricco né aristocratico, non pervertito dall'avarizia, come i «litterati di questa lingua (italiana)», che quindi «acquisterà la lettera per lo suo uso» e non "per guadagnare denari o dignitate» (Conv., I, ix, 2-3). Ciò spiega che il suo raggio d'azione sia non la cristianità nel suo complesso (né l'umanità in generale), ma l'Italia, della quale egli sarà salute, cioè salvezza, in un senso che non ha nulla a che vedere con la «armorum probitas» della Rota Virgilii, e che implica invece quella redenzione etica, estetica e metafisica che è tema caratteristico della poesia dantesca. D'accordo con il programma politico-linguistico del $1^{\circ}$ trattato del Convivio, e del De Vulgari, il veltro sarà salvezza per gli Italiani, intesi come una collettività culturale omogenea e distinta dalle altre, poiché fornirà loro la nuova lingua di cultura nella quale potrà esprimersi la loro identità etnica, ossia l'italiano. ${ }^{47}$ Come Dante ha dimostrato nelle due opere, il conflitto linguistico volgare-latino riflette direttamente il conflitto politico fra una concezione laica dello stato e una concezione ecclesiastica, e l'Italia può avere identità politico-culturale (all'interno dell'Impero, ma comunque distinta dagli altri regni nazionali della cristianità), solo se i suoi intellettuali adotteranno il volgare italiano e i valori laici che esso esprime poeticamente. L'avanguardia intellettuale che dovrà fare l'Italia dando forma alla sua lingua è rappresentata da poeti, dei quali il veltro è figura allegorica nella quale Dante ha proiettato la propria coscienza poetica, proponendosi così come modello della nuova classe di intellettuali di cui l'Italia ha bisogno per esistere come nazione. ${ }^{48}$ Certo, Virgilio definisce l'Italia a partire dalla fondazione mitica di

46. Si consideri che Dante è abbastanza severo nei confronti dell'uso venale della filosofia: «Né si dee chiamare vero filosofo colui che è amico di sapienza per utilitade, sì come sono li legisti, [li] medici e quasi tutti li religiosi, che non per sapere studiano ma per acquistare moneta o dignitade; e chi desse loro quello che acquistare intendono, non sovrasterebbero a lo studio" (Conv., III, xi, 10).

47. Sul valore etnico che ha qui l'Italia, d'accordo con tutta la restante opera dantesca, cfr. Antonino Pagliaro, Il proemio, in Ulisse..., p. 43-53.

48. Non può coincidere, quindi, con il veltro il "cinquecento diece e cinque» di Purg., XXXIII, 43 , che, in quanto «reda della aguglia», allude necessariamente ad un politico (con simbo- 
Roma, così come viene celebrata nell'Eneide. Ma confondendo ed alternando vincitori troiani e vinti italici, egli allude molto più alla Italia moderna potenzialmente unificata attorno ad una corte e ad una lingua (così come la pensa Dante), che non al territorio a partire dal quale Roma avrebbe creato il suo impero. E definendosi mantovano (come nel VI del Purgatorio), mette in risalto, più che la sua latinità, la sua italianità, indipendente dalla lingua che usa (che comunque, per il fatto di essere latino dell'età classica, è esente dalla prostituzione letteraria dei chierici).

In questa evocazione dell'Italia come ambito della azione redentiva del veltro, sono del resto presenti simboli generici troppo evidenti e troppo legati alla esperienza poetica dantesca per non pensare che nel veltro il poeta abbia inteso rappresentare se stesso. Innanzitutto l'umiltà dell'Italia, lontanissima dal senso che l'aggettivo ha nell'Eneide ("pianeggiante») ed invece vicinissima alla immagine positiva di donna che Dante e Cavalcanti avevano descritto nella loro lirica. In Guido (Chi è questa che vèn, 7) «donna d'umiltà» è l'espressione con cui viene indicata l'amata, ed a lei sono connesse, in rima, come qui, la vertute e la salute dell'amante. In Dante umiltà e salute sono le prerogative tipiche di Beatrice, «benignamente d'umiltà vestuta", in Tanto gentile, e "donna della salute», nella Vita Nuova. L'immagine dell'Italia (qui, come poi nel VI del Purgatorio) è chiaramente costruita secondo la simbologia femminile elaborata dal poeta liricamente, cioè come supremo oggetto di desiderio da parte di un io poetico maschile.

In effetti, l'allusione all'Italia determina drasticamente, nella geografia e nella storia, un paesaggio finora solo simbolico ed onirico, ed indica con precisione autobiografica i termini, fisici e ideali, del territorio in cui il Poema aspira ad essere letto: si tratta «delle parti quasi tutte a le quali questa lingua si stende», per le quali il poeta è andato "peregrino, quasi mendicando», sferzato «dal vento secco che vapora la dolorosa povertade» (Convivio, I, iii, 4-5). Ma questa brusca caduta del simbolico nel reale ha anche l'effetto di capovolgere i valori assegnati ai simboli sessuali. Il femminile è ora, nella prospettiva originalissima della propria poesia, il valore assolutamente positivo che introduce un generale cambiamento di direzione della storia, aprendo le porte della modernità a un nuovo sistema di valori, coincidenti con quelli che Dante è andato elaborando nelle opere anteriori, e che hanno nella donna e nel femminile (cioè Beatrice) il loro simbolo più elevato.

Tale femminilità redentiva si precisa subito in una immagine di strordinaria efficacia evocativa, per la densità delle allusioni poetiche e culturali che contiene: la "umile Italia» di cui il veltro sarà salute ha già avuto dei martiri, ma il primo di loro ad essere nominato è «la vergine Camilla». Fra gli eroi ricordati da Virgilio, quella più significativa (l'unica del gruppo che apparirà poi nel Limbo, a stretto contatto con Cesare) è dunque una donna, la cui vergi-

logia numerologica di tipo apocalittico). Ma intercorre, fra i due passaggi, la riflessione sullo stato che sfocia nella composizione della Monarchia. 
nità indica la purezza dei costumi, in antitesi con la lupa che si ammoglia con molti, ed in consonanza solo parziale con la madre di Cristo, alla quale la accomuna la verginità, ma dalla quale la distingue il fatto di essere una guerriera, e di contribuire alla salute degli Italiani morendo. È probabile che qui Dante stia di nuovo pensando a Beatrice, che solo da morta potrà salvarlo, come Cristo, che salva l'umanità morendo. La Camilla dantesca potrebbe essere definita come la controfigura epica di Beatrice; essa rappresenta, nell'universo poetico virgiliano, la prefigurazione della donna che, morendo, salva Dante, aprendo alla sua immaginazione e al suo desiderio lo spazio metafisico della trascendenza.

Ond'io per lo tuo me' penso e discerno
che tu mi segui, e io sarò tua guida,
e trarrotti di qui per loco etterno,
ove udirai le disperate strida,
vedrai li antichi spiriti dolenti,
ch'a la seconda morte ciascun grida;
e vederai color che son contenti
nel foco, perché speran di venire
quando che sia a le beate genti.
A le quai poi se tu vorrai salire,
anima fia a ciò più di me degna:
con lei ti lascerò nel mio partire;
ché quello imperador che là sú regna,
perch'i fu' ribellante a la sua legge,
non vuol che 'n sua città per me si vegna.
In tutte parti impera e quivi regge;
quivi è la sua città e l'alto seggio:
oh felice colui cu' ivi elegge!».

La descrizione di tale itinerario alternativo (un «loco etterno», cioè la zona d'ombra del reale, la geografia della morte che i due poeti dovranno attraversare per ricostruire quel rapporto con la trascendenza che i litterati hanno spezzato per la loro avidità) ha senso, nell'ottica del simbolismo dei generi, nella distinzione delle due guide. Alla seconda si allude con una espressione genericamente vaga, "un'alma", che crea, però, intorno a lei un'attesa che rende ancora più clamoroso il trionfo del femminile del canto seguente. Le tre donne che dal Paradiso si preoccupano, in Inf. II, della ventura di Dante corrispondono, infatti, sullo stesso piano generico ma con inversione di segno, alle tre fiere che gli impediscono il cammino. E se Virgilio è il razionalismo della poesia ("Tratto t'ho qui con ingegno e con arte», Purg., XXVII, 130), Beatrice è, sullo stesso piano simbolico ma con inversione di genere, il teologismo della poesia, cioè, come si è cercato di mostrare in altra occasione, ${ }^{49}$ il compito modernamente assegnato al linguaggio di umanizzare il trascendente, trasformandolo 
in esperienza personalmente vissuta, compito che lei porta a termine in quanto fu ed è ancora, dopo morta, fonte di piacere e oggetto di desiderio per il poeta, («lo tuo piacere omai prendi per duce», ibid., 131). La sua femminilità è intrinseca al suo ruolo di guida e maestra, ed anzi ne è aspetto sostanziale, poiché è grazie ad essa che la conoscenza, nel suo rango dottrinale più elevato, si traduce finalmente nella parola poetica che la umanizza. Con Beatrice il femminile irrompe nel discorso culturale italiano ed europeo, non come astratta personificazione ma come a priori espressivo, smentendo il teologismo tradizionale nel suo fondamento generico-antropologico, cioè nella pregiudiziale antidottrinale che grava sulla donna, secondo la dura ingiunzione paolina (I Tim., 2, 11-15):

Mulier in silentio discat cum omni subiectione. Docere autem mulierem non permitto, neque dominari in virum: sed esse in silentio. Adam enim primus formatus est: deinde Eva: et Adam non est seductus: mulier autem seducta in praevaricatione fuit. Salvabitur autem per filiorum generationem, si permanserit in fide, et dilectione, et santificatione cum sobrietate.

Se si tiene conto della cultura teologica antica (che questo frammento perfettamente riassume nel suo rigore misogino), ${ }^{50}$ ancor più improbabile del viaggio di un vivo nell'al di là, è il fatto che a guidarlo, insegnando, sia un essere umano di sesso femminile. ${ }^{51}$

«Poeta antico» (Inf., X, 121), Virgilio fu ribellante alla legge di Dio (benché ne intuisse oscuramente la presenza) e quindi gli è precluso l'accesso alla dimensione spirituale dell'al di là (la cristiana Civitas Dei), nella quale Dante sarà guidato dalla donna che ha ispirato una parte fondamentale della propria poesia. Il salto cronologico dall'antico al moderno implica un radicale cambiamento di prospettiva ermeneutica: antichità e modernità non si oppongono più solo sul piano religioso (paganesimo vs. cristianesimo); fra di essi si è aperta una divaricazione linguistica (latino vs. volgare) e generica (maschile vs. femminile) che in certo modo neutralizza quella opposizione (l'unica pertinente, in una prospettiva clericale). Mentre nella cultura antica il sistema simbolico dei generi prevede la supremazia del maschile sul femminile (ciò che la letteratura latina manifesta attraverso il primato dell'epica), nella cultura moderna esso adombra la supremazia del femminile sul maschile (ciò che la

50. Il culto mariano temperò in parte, come è noto, tale misoginia. Si osservi però che nella Vergine viene esaltata, con paradosso dogmatico, proprio la maternità, cioè l'unica funzione positiva che la teologia tradizionale attribuisce alla donna (che, in Paolo, «salvabitur per filiorum generationem»).

51. Confondere Beatrice con le antiche allegorie femminili della sapienza (come nelle letture mistico-esoteriche di Dante) significa trascurare la sua principale novità storica, e cioè l'istanza espressiva femminile e moderna di cui lei è veicolo (sull'idea di lingua materna in Dante e nel dibattito umanistico-rinascimentale, cfr. il mio La donna come alterità linguistica, in Sergio ZATTI (a cura di), La rappresentazione dell'altro nei testi del Rinascimento, Lucca: Pacini Fazzi, 1998, p. 13-32). 
letteratura italiana delle origini manifesta attraverso il primato della lirica e l'emarginazione dell'epica). Indicando in Beatrice una guida di rango più elevato («anima più di me degna»), Virgilio sancisce sul piano ideologico tale trasformazione. Le dicotomie generiche fin qui evidenziate scambiano infatti i loro valori, innanzitutto quella «basso / alto», poiché è Beatrice che, in quanto donna desiderante e desiderata, permetterà a Dante di salire, cioè di accedere poeticamente alle «beate genti»; 52 e poi quella «ombra / luce», che prima, nella polarità «valle - colle», subordinava il femminile al maschile, mentre ora, nella polarità «terra - cielo», subordina il maschile al femminile. E lo stesso vale, implicitamente, per tutte le altre.

Il nuovo sistema simbolico generato dalla inversione dei valori non è però dualistico, poiché il femminile supera il maschile senza contrapporsi ad esso. ${ }^{53}$ Fra i due generi sessuali e le rispettive simbologie esiste un rapporto di complementarietà: bisogna attraversare la regione fisica e maschile (sublunare) della trascendenza («l'erte vie e l'arte» di Purg., XXVII, 132) per accedere alla regione, spirituale e femminile, della luce. Un nuovo dualismo si profila, invece, come s'è visto, sul piano della coscienza storica, nella opposizione di antico (assiologicamente maschile) e moderno (assiologicamente femminile). Ma qui sono implicate le componenti più personali della riflessione dantesca sulla letteratura, e cioè la novità e la modernità della propria poesia: lo scatto trascendentale che permette a Dante di superare poeticamente i limiti dell'universo fisico e di secolarizzare lo spazio metafisico, è possibile solo in virtù di quel culto del femminile con cui egli ha spiritualizzato l'eros e che è stato il tema dominante di tutta la sua ricerca anteriore alla Commedia. Fulminea ricapitolazione di tale ricerca, citata praticamente in ogni suo versante, il I canto dell'Inferno presenta il Poema come l'approdo di una esplorazione che, partendo dalla lirica, ha interrogato la cultura letteraria investigandone tutti gli aspetti,

52. Lo stesso schema si ripete nell'ultima parte del viaggio, in cui S. Bernardo preannuncia a Dante la successiva e superiore mediazione di Maria (Par., XXXI, 100-102: "E la regina del cielo, ond'io ardo / tutto d'amor, ne farà ogne grazia, / però ch'i' sono il suo fedel Bernardo").

53. Tale inversione di valori si avverte nelle parole con cui viene celebrata la prima apparizione di Beatrice a Dante in Vita Nuova, II, 4: «lo spirito della vita... disse queste parole: «Ecce deus fortior me, qui veniens dominabitur michi». La frase allude, in prima istanza, al dominio che il desiderio esercita, attraverso Beatrice, sulla vita del poeta (dominio che è interno alla mente, ed implica quindi l'adesione dell'io). Il testo però è infarcito di risonanze scritturali (su cui cfr. Domenico De RoberTis, D.A., Opere Minori..., t. I, p. I, p. 31), fra le quali segnalerei la condanna di Eva in Genesi, 3, 166: «sub viri potestate eris et ipse dominabitur tui", che mostra bene come l'adesione dell'io maschile al principio del desiderio implichi il capovolgimento del rapporto di potere e dominio fra i generi sancito dalla cultura patriarcale antica. Tale capovolgimento ha quindi un'origine storica precisa: esso si produce nel momento in cui l'amore di un sesso verso l'altro diviene il fondamento della letteratura, naturalizzando la cultura attraverso il linguaggio. D'altra parte la reversibilità del desiderio (in quanto soggetto desiderante la donna si espone al dominio di una immagine maschile) neutralizza il fondamento generico del dualismo antico, che sopravvive modernamente solo come astratto paradigma filosofico (razionale vs. irrazionale). 
ma senza mai abbandonare l'oggetto che fin dal principio l'ha ispirata, il culto, appunto, ossessivo ma anche redentivo, del femminile. Ecco allora che il desiderio, che la poesia moderna adotta come unico ed assoluto principio ispiratore (a norma di Vita Nuova, XXV e Purg., XXIV e XXVI), può divenire il fattore storicamente originale che capovolge il rapporto fra i generi sessuali ed il loro stesso significato simbolico, permettendo per la prima volta ad un essere umano, in quanto poeta, di varcare la soglia della trascendenza:

E io a lui: «Poeta, io ti richeggio per quello Dio che tu non conoscesti, acciò ch'io fugga questo male e peggio, che tu mi meni là dov'or dicesti, sì ch’io veggia la porta di san Pietro e color cui tu fai cotanto mesti». Allor si mosse, e io li tenni dietro. 